\title{
Animal and human models to understand ageing
}

Hayley Lees, Hannah Walters and Lynne S. Cox ${ }^{1}$

Department of Biochemistry, University of Oxford, South Parks Road, Oxford, OX1 3QU, UK

1. Author for correspondence: Iynne.cox@bioch.ox.ac.uk

\begin{abstract}
Human ageing is the gradual decline in organ and tissue function with increasing chronological time, leading eventually to loss of function and death. To study the processes involved over research-relevant timescales requires the use of accessible model systems that share significant similarities with humans. In this review, we assess the usefulness of various models including unicellular yeasts, invertebrate worms and flies, mice and primates including humans, highlighting the benefits and possible drawbacks of each model system in its ability to illuminate human ageing mechanisms. We describe the strong evolutionary conservation of molecular pathways that govern cell responses to extracellular and intracellular signals and which are strongly implicated in ageing. Such pathways centre around insulin-like growth factor signalling and integration of stress and nutritional signals through mTOR kinase. The process of cellular senescence is evaluated as a possible underlying cause for many of the frailties and diseases of human ageing. Ageing arising from systemic changes that cannot be modelled in lower organisms are also considered, as these require studies either in small mammals or in primates including humans. We also touch briefly on novel therapeutic options arising from a better understanding of the biology of ageing.
\end{abstract}

Key Words: Ageing; progeroid syndromes; yeast; C. elegans, Drosophila; mTOR 


\section{Introduction}

Ageing can be biologically defined as a loss of cellular, tissue, organ and system function with increasing chronological time, leading ultimately to death. It involves gradual and often stochastic molecular changes leading to loss of homeostasis that predisposes to the diseases and frailty characteristic of older age, such as sarcopenia, osteoporosis, lipodystrophy, cardiovascular disease, diabetes, cancer and dementia. Aging thus carries a huge personal cost of physical and often mental decline, coupled with emotional costs to the affected individuals and their families, and financial pressures in providing suitable social and medical care. Estimated age-related healthcare costs in the UK are currently $6.8 \%$ of GDP (in the region of $£ 100 \mathrm{bn} p a)$, and this is set to rise with an increasingly ageing population [1]. Thus understanding ageing, with the promise of developing new ways to delay or prevent the onset of age-related frailty and disease, or treat specific age-related conditions more rationally and more successfully is now a major research focus in many developed countries.

Conserved hallmarks of ageing have recently been described [2]. These generally cover either loss of function or dysregulation of normal cellular activities including: cell communication, genome stability, telomere maintenance, protein homeostasis, mitochondrial function, nutrient sensing and epigenetic control of gene expression. These changes are likely to result in two additional hallmarks, stem cell exhaustion and cellular senescence.

Senescence is a state of essentially irreversible cell division arrest, whilst retaining cell viability [3]. Marked changes in morphology (enlarged size, irregular cell shape, prominent and sometimes multiple nuclei, accumulation of mitochondrial and lysosomal mass, increased granularity and highly prominent stress fibres (e.g. [4]) are accompanied by shifts in metabolism, including a failure of autophagy [5]; detecting senescent cells in vitro and in tissue and organs is possible using either chromogenic or fluorescent substrates of the enzyme $\beta$ galactosidase, that is highly elevated and acts at relatively high $\mathrm{pH}(\sim 6)$ in senescent cells [6]. In addition, there is often a shift to glycolytic metabolism away from oxidative phosphorylation [7], despite a marked increase in mitochondrial mass and markers of mitochondrial activity [8]. Lysosomal pH may rise as a consequence of proton pump 
failure, leading to an inability to rid the cell of damaged macromolecules - this may be a cause of the failure of autophagy in senescent cells and the granularity observed microscopically [9]. Molecular drivers of senescence and the impact of senescent cells on ageing have been reviewed elsewhere [10] [11] [12] and are also considered briefly in relevant sections below.

Studying ageing in humans is a challenging task, both because of the sheer time frames involved in any longitudinal study, and also the ethical difficulties in testing hypotheses experimentally. In order to understand how ageing occurs at the cellular and molecular level, and to dissect the contribution of individual genes and epigenetic phenomena to ageing, the use of model organisms has proven invaluable. In this review, we discuss the various models that have been particularly informative in ageing studies, touching on yeast, worms, flies, mice, non-human primates and humans (both those undergoing 'normal' ageing and patients with premature ageing syndromes). We assess how recent technical advances have allowed the lessons learned in model organisms to be compared with findings in humans, for testing and refinement of possible anti-ageing therapies.

\section{What constitutes a good ageing model?}

Though ageing is a widespread phenomenon in biology, it is by no means universal: lobsters [13] and hydra [14] show no loss of tissue integrity or reproductive capacity with increasing chronological time, while the North Atlantic Quahog (a hard shelled clam) can live to at least 400 years with no obvious signs of 'ageing' [15]. Salamanders retain regenerative capacity over long periods of chronological time [16] and acute cell senescence is required during regeneration [17]; it is possible that senescence may initially have arisen as a developmental and/or wound healing mechanism that has only recently in evolutionary time been co-opted as a tumour suppressor mechanism with ageing as a side-effect [18]. A model to study ageing must therefore fulfil the following criteria: it must have measureable phenotypes of ageing and the underlying biochemical mechanisms of ageing must be conserved with humans. Ideally, the chosen organism must be relatively easily manipulated under laboratory conditions (e.g. small and cheap to grow in large numbers), and further ethical consideration should be given to working with sentient higher organisms. Widely studied model organisms in ageing research are therefore brewer's yeast (Saccharomyces 
cerevisiae), the nematode Caenorhabditis elegans, and the fruit fly Drosophila melanogaster, though mice and non-human primates are also studied (Table 1).. In addition, the utility of models lies not only in their genetic relevance to humans and their ease of manipulation in the lab, but also the degree of curation of the resources that support such analyses [19-21], including access to organisms of known genotype plus relevant cDNA constructs via centralised resource centres (Yeast Genetic Resource Center (YGRC); Caenorhabditis Genetics Center (CGC); Drosophila Genomics Resource Center (DGRC)), which both saves experimental time and ensures consistency between different laboratories. It is becoming increasingly clear for work on these models that there has been very strong evolutionary conservation of key biochemical pathways that influence ageing. In choosing an appropriate model, is important to bear in mind the key aspects of ageing to be studied in order to decide whether the model has sufficient complexity in terms of tissue and organ structures in order to correctly model chosen aspects of ageing such as neurodegeneration or cardiovascular disease.

\section{Yeast}

The simplest organism used in studies of eukaryotic ageing is the brewer's yeast, Saccharomyces cerevisiae. This single-celled organism shares a significant number of important genes with humans, such that lessons from yeast cell division have been vital in underpinning our understanding of cell cycle control and have led to novel cancer therapies. Because S. cerevisiae is haploid, genotype to phenotype analysis is straightforward, and ageing can be assessed as either replicative life span (RLS, the number of cell divisions a 'mother' cell can undergo) or chronological life span (CLS, essentially how long a yeast cell can remain viable in a post-mitotic state). The simplicity of the single yeast cell allows for rapid phenotypic characterisation of the impact of mutations thought to be involved in human ageing; for example, expression of mutant human genes encoding either $\alpha$-synuclein (in human Parkinson's disease) or huntingtin (Huntington's disease) results in yeast cell killing, supporting the notion that toxicity due to protein aggregates contributes to human neurodegeneration during ageing [22-24].

An unbiased screen testing over 500 single gene deletions for impact on RLS, [25] identified two kinases, TOR1 and its downstream target Sch9, which when deleted increased yeast RLS 
[26]. TOR kinase is so named as it is the mechanistic target of an antibiotic rapamycin (mTOR); both mTOR and Sch9 are emerging as both highly conserved and critically important in ageing across all eukaryotes so far analysed A potent modifier of lifespan is caloric restriction: yeast cells grown in low nutrient conditions show elevated longevity, and this appears to be mediated through the TOR1 signalling pathway [27]. Further molecular analysis, such as determination of the yeast phosphoproteome with and without rapamycin treatment has led to identification of critical downstream targets of mTOR that may also be involved in regulating ageing - lower phosphorylation of GCN2 (eIF2 kinase) and the RNA binding protein LHP1 and increased phosphorylation of PKA-inhibitor BCY1 [28] together suggest that nutrient sensing (leading to increased protein translation and cell growth) may promote ageing. Integrating nutritional status with cellular stress requires additionally TOR modulation of a forkhead transcription factor, FOXO. The lessons from yeast have proven particularly valuable in understanding how stress and ageing may be linked in higher organisms (see below). Other yeasts such as Schizosaccharomyces pombe have until recently been less well studied in relation to ageing because their cell division is symmetrical so the technique of counting bud scars from asymmetric division is simply not possible. However, advances in micromanipulation and microfluidics have led to the development of a replicative lifespan screening protocol applicable to S. pombe [29].

Overall, yeasts have the benefit of direct genotype to phenotype analysis, extremely rapid generation time (3h), small genome, a large resource of characterised mutants, growth on both solid and liquid media, and scalability such that cultures can contain billions of individual cells. This is particularly valuable when considering high-throughput screening and has recently been exploited to assess genetic interactions that may be important in ageing [30]. However, the very features that make yeast amenable to rapid and straightforward culture also pose significant drawbacks, particularly in attempting to study aspects of ageing that are a consequence of complex intercellular interactions in metazoans. While yeast cell ageing has been likened by some to human cellular senescence, yeast lack the inflammatory intercellular signalling characteristic of senescent cells that is likely to cause deleterious effects on neighbouring cells in humans. 


\section{Worms}

Since C. elegans was first described as a model for studying ageing nearly 40 years ago [31], research on genetic and environmental manipulations which can alter lifespan and ageing has shown some remarkable insights into how single genes can have very marked impacts on longevity and healthspan. The transparent $\sim 1 \mathrm{~mm}$ long $C$. elegans is easy and cheap to grow, and exists primarily as self-fertilising hermaphrodites which lay around 300 eggs each, allowing large numbers of genetically isogenic animals to be generated over a very short time frame of three days. The short, largely invariant adult lifespan of $C$. elegans (average $\sim 17$ days at $20^{\circ} \mathrm{C}$ ), coupled to the fact that the entire genome has been sequenced and annotated [32] has allowed the identification of over 200 genes and regimes that affect lifespan in the nematode. Furthermore, C. elegans provides an invaluable system for comprehensive reverse and forward genetic screens, owing to the existence of an RNAi library covering roughly $80 \%$ of the worm's genes, and the ease of genetic manipulations including the ability to generate transgenic strains, including using recent gene-targeting approaches such as CRISPR. The marked evolutionary conservation of not only genes (the genome of $C$. elegans contains homologues of approximately two-thirds of all human disease genes [33]) but also biological pathways between worms and humans allows findings in this relatively simple organism to be applied to the understanding of human ageing.

A major breakthrough in field of the biology of ageing was made in C. elegans - namely, the combined findings from Klass [34]) and then Johnson [35] demonstrating that individual genes can modulate lifespan. These studies challenged the existing dogma that ageing was an inevitable, fixed process of wear and tear. The first such gene to be identified was age-1, which encodes a phosphatidyl inositol 3-kinase (PI3-kinase) [36]. Isolation of further mutants demonstrated that insulin-like growth factor signalling (IIS), which is conserved across phyla, is a major regulator of organismal ageing [37] [38] . Functional DAF-16, the worm FOXO transcription factor, has been demonstrated to be required for the longevity of many of the lifespan enhancing mutations [39]; [40]. Interestingly, human population studies have revealed an association between single nucleotide polymorphisms (SNPs) in human FOXO3 and human lifespan extension [41]; [42] [43]; [44]) and the strength of the association appears to increase with age [45]. Therefore, FOXO3 appears to be a candidate 
longevity gene in humans and thus demonstrates how work in the humble nematode worm can inform on the biology of human ageing. Subsequent work using $C$. elegans has shown the importance of several other evolutionarily conserved pathways and processes in ageing, namely caloric restriction, mitochondrial pathways, energy metabolism, endocrine signalling and signal transduction, the stress response, protein translation and gene expression [46]. Despite their marked similarities to ageing pathways in so many ways, worms have not been studied in the context of cellular senescence. It is likely that this is because they are generally considered post-mitotic in the adult state, though both mitotic and meiotic cell division occur in the adult gonad making them amenable to studies of factors that impact on proliferative capacity and possibly senescence.

C. elegans can also be utilised to understand other aspects of ageing including population and evolutionary studies, hormetic treatments and environmental manipulations including more detailed studies on the effect of diet (encompassing the microbiome) and, excitingly, models of age-related diseases [47]. Elegant examples of this are worm models of Alzheimer's and Parkinson's disease [48-50]. Worms can be challenging to work with in terms of drug interventions, requiring very high drug concentrations in order to penetrate the cuticle though this can be overcome to some extent using cuticular bus mutants [51]. This combined knowledge is being used to establish drug screens for compounds which not only extend lifespan, but importantly, health-span, by delaying the ageing process.

\section{Flies}

The fruit fly Drosophila melanogaster is widely used in ageing research, having a relatively short lifespan of 40-50 days and a generation time of only 9 days (egg to adult) at a temperature of $25^{\circ} \mathrm{C}$. Having a fully sequenced and annotated genome simplifies genetic analysis, as do the tools developed for genetic manipulation (such as P element and piggyBac transposons) and phenotypic markers for analysis (e.g. eye colour and obvious changes to adult wing hairs). It is also possible to directly assess fly homologues or orthologues of genes implicated in human disease as these have been curated, originally in the Homophila database [52] and now directly through Flybase. Isogenic fly lines can readily be obtained through crossing. It is also relatively straightforward to breed flies in large numbers and to manipulate them both genetically and via changing their environment (e.g. food levels and composition, stressors such as temperature or ROS generators). Flies have 
distinct developmental stages including embryonic, larval (with 3 distinct larval instars), pupal (where major tissue reorganisation occurs) and adult stages. The timing of each stage is well characterised for wild type flies under lab conditions, as is the lifespan and certain traits that indicate ageing, such as distance climbed in a fixed time.

While anatomically very distinct from mammals, flies do have certain tissues that serve similar functions - for example, the fat body functions in a role akin to the mammalian liver in regulating energy metabolism. Because flies have a reasonably complex nervous system, phenotypes that are relevant in humans can be usefully modelled e.g. the shaker phenotype that occurs on mutation of the Sh gene (which encodes a voltage-sensitive potassium channel in the nervous system) models defects in the homologous human KCNA3 gene responsible for some forms of multiple sclerosis. Inflammation has been closely linked with early ageing phenotypes in humans and this can be modelled to some extent as the fly has innate immunity mediated through Toll-like receptors and NF kappa B signalling. While cellular senescence per se has not been reported in flies, stem cell exhaustion in the ovary has been characterised (reviewed by [53]).

Flies, like worms and yeast, are highly sensitive to nutritional status through insulin-like growth factor signalling (IIS) and mTOR-dependent signalling (involving mTORC1 and S6k, the fly homologue of yeast Sch9). For example, severe dietary restriction in flies increases lifespan; the importance of insulin signalling is emphasised by the finding that flies homozygous mutant for the insulin receptor substrate, Chico, show lifespan increases of nearly $50 \%$ [54]. Moreover, modification of the mTORC signalling pathway, through mTOR mutation [55], rapamycin feeding [56] and S6K mutation [55], greatly extends fly lifespan. Thus fly genetics can inform studies in higher organisms. However, factors identified in other screens to identify genes that regulate longevity have revealed factors such as Methuselah, a G-protein coupled receptor (GPCR) [57] which do not appear to have mammalian homologues.

A major limitation in using flies is the difficulty in assessing phenotypes of ageing other than lifespan, which is intensive to study in terms both of labour and time. One recent advance in this regard is the demonstration of age-related increased gut leakiness detectable by uptake of an inert blue due administered in the diet; because of the obvious blue colour of the aged 
flies, this has become known as the 'Smurf assay' [58]. A further limitation is the difficulty of administering pharmacological or other agents to screen for improvements in longevity. Dietary administration to larvae is possible [59], though drug doses are generally significantly higher than those used in mammals (e.g. camptothecin at $500 \mathrm{nM}$ has no impact on viability of wild type flies [60] while the $\mathrm{IC}_{50}$ in human cells is less than $50 \mathrm{nM}$ ). Drugging early stages of development is even more challenging due to the impervious chorion and waxy layers protecting the early embryo, though recent advances in embryo permeabilisation look promising [61].

\section{Rodents}

While yeast, worms and flies have proven invaluable in studying aspects of ageing, particularly those dependent upon single gene mutation or well defined biochemical pathways, they have limitations in terms of modelling system-level changes on ageing. For this, mammalian models have utility. Mice (Mus musculus) are widely used in ageing research, though until recently inbred mouse strains were chosen because of genetic homogeneity. These mice (e.g. C57BL/6) have a lifespan of about 2 years compared with genetically heterogeneous wild type mice (maximum lifespan of $\sim 4$ years), and are less likely to reflect the genetic diversity of human populations. Transgenic mice bearing specific introduced genetic changes can be generated by manipulating the genome of embryonic stem cells that are then implanted into the developing blastocyst. Genome editing originally relied on low efficiency homologous recombination techniques but more recently, targeted nucleases including zinc finger or TALENS nuclease, or the CRISPR-Cas9 gene editing systems have proven of value. To overcome potential problems with knockouts that impact on development, it has been possible to generate conditional knockouts using the Cre-Lox system so that genes implicated in ageing but also essential for development can be inactivated post-development to assess their function in adult life. Databases now exist mapping changes in mouse tissues with age including the AGEMAP (Atlas of Gene Expression in Mouse Aging Project) gene expression database [62], while ageing researchers now collaboratively share tissues from aged mice through the ShARM resource [63], which minimises the use of mice in ageing research while maximising the outputs from any studies that have already taken place. 


\section{Metabolic regulation in rodent ageing}

Some of the earliest studies on ageing were carried out in brown rats fed a very limited diet [64]; such caloric restriction led to remarkable increases in lifespan. Follow-on studies then included pharmacological interventions showing that the mTORC1 pathway and possibly also the sirtuins are involved in the lifespan-boosting effects of caloric restriction (reviewed in [65]). Consistent with studies in lower organisms suggesting a critical role for mTORC1 in ageing, rapamycin fed to mice in late middle age led to increases of between $9-13 \%$ in lifespan in a highly powered three-site study through the Interventions Testing Program (ITP[66]); notably resveratrol under similar conditions did not impact on longevity [67].

\section{Modelling neurodegeneration in mice}

Mice show complex behaviours, so that neurological degeneration associated with ageing, including Alzheimer's and Parkinson's disease, can be modelled at the genetic and molecular level and also in terms of measuring changes in cognition. Tests of pharmacological modifiers of dementia have highlighted rapamycin, the mTORC1 inhibitor, as potentially beneficial in mice with Alzheimer's and Parkinson's disease [68-70]; such studies provide compelling neuroanatomical and behavioural evidence that interventions can work in complex nervous systems and should provide sufficient pre-clinical validation to support future testing in humans showing early cognitive decline.

Mice also have both innate and adaptive immune systems highly similar to those in humans, so the effects of immune senescence can be modelled. Interventions that may improve immunological outcomes in ageing have been tested - for example, spermidine has been shown to improve immunity in aged mice [71].

\section{Differences in triggers of senescence between mice and humans}

Rodents generally (but not universally- a marked exception is the naked mole rat) invest in reproduction over maintenance of the soma, and this may not reflect the trade-off between fecundity and longevity that occurs in primates including humans. Additionally, at the molecular level mice show some significant differences in ageing mechanisms: mouse telomeres are five to ten times longer than human telomeres though mice live for only 2-4 years compared with $~ 80$ years for humans; mice die well before their telomeres have reached critically short lengths even though this is a trigger of cellular senescence in human 
cells (see below). . The classic early ageing features of Werner syndrome in humans are not recapitulated in mice mutant for the WRN gene, even though WRN is very highly conserved between mice and men. However, if mice lacking telomerase activity (terc ${ }^{-/}$) are crossed with WRN null mice, after 5-6 generations, they do now show classical Werner syndrome phenotypes, suggesting that telomere shortening is required to unmask such ageing phenotypes - or conversely that WRN loss per se does not cause aging if telomeres are intact. Interestingly this hypothesis arising from mouse studies has been tested in human cells by over-expressing telomerase in patient-derived WS cells; these no longer senesce prematurely but continued to proliferate even though WRN activity was missing [72]. One potential conclusion is therefore that the major role of WRN is to protect cells from the impact of shortened or uncapped telomeres.

\section{Cell senescence in mice}

Mouse cells undergo cellular senescence in response to many of the triggers also known to drive senescence in human cells, including DNA damage and high ROS. Induction of senescence in mice relies heavily on the p16 cyclin kinase inhibitor CDKN2A, while p21 CDKN1A may be more important in some cell types in humans [73]. However, the role of the tumour suppressor protein p53 appears conserved in mediating senescence, as mice expressing a 'super-p53' that is highly active undergo premature ageing [74].

Cellular senescence can be conceptualized as a protective mechanism to prevent the proliferation of damaged or dysfunctional cells. It has been suggested that it plays a role in ageing: senescent cells accumulate with age in a range of tissues and organs and may disrupt tissue function as well as causing inflammation [11]. However, direct evidence that senescent cells drive ageing and the progression of age-related diseases was lacking until a group of molecular biologists at the Mayo Clinic in Minnesota developed a sophisticated transgene INK-ATTAC system whereby administration of a drug selectively eliminated cells expressing p16Ink4a in progeroid BubR1 mice. Elimination of senescent cells delayed the onset of age-related pathologies such as hunchback (kyphosis) and cataracts. Further, clearance of senescent cells late in life could even attenuate the progression of alreadyestablished disorders [75]. This has recently been verified in wild type mice, where senescent cell clearance led to lifespan extension and attenuation of age-related deterioration [76]. These exciting results provide direct evidence for a causative role of 
senescent cells in ageing and in the progression of age-related diseases, as well as suggesting that their removal could be beneficial.

These genetic experiments provide proof-of-concept that senescent cell removal can be beneficial, but achieving the same using small molecules would make removal of senescent cells a viable therapeutic option for treating age-related diseases in humans. A potent senolytic drug ABT263 that selectively kills senescent cells was identified in a screen by Chang et al [77]. ABT263 is an inhibitor of BCL-2 and BCL-xL, two anti-apoptotic proteins; oral treatment of old mice with ABT263, resulted both in depletion of senescent cells and in tissue rejuvenation., while in an independent study, a combination of two different senolytics (dasatinib and quercetin) depleted senescent cells of aged mice and rapidly rejuvenated the mice [78].

While laboratory mice provide excellent models for some aspects of ageing, inbred strains age very quickly, and additionally invest less than humans in somatic maintenance so they have a much higher cancer incidence than in man. Since senescence is thought to have evolved (at least in part) as a tumour suppressor mechanism, it is likely that studies of senescence in mice may not fully mimic the process or its consequences in humans. Studies on long lived rodents have therefore been initiated in naked mole rats, following the finding that these social rodents have an extraordinarily long lifespan of $\sim 30$ years and an extremely low cancer incidence [79]. Genome analysis suggest that a modified form of p16 CDKN2A may be important in this context [80], as may very long chain hyaluronic acid [81]. Which these two extremes of rodent longevity (lab mice and naked mole rats) most closely models the process of ageing in humans is currently moot, though it may be that aspects of primate ageing (especially higher cognitive functions) differ from those in rodents and therefore that primates may be the best model to study primate ageing.

\section{Dogs}

Interventions developed through testing on lower organisms are not always successful when applied to humans, possibly because of the failure to take into account anatomical and organ system differences as well as genetic heterogeneity. A recent trial has been established in pet dogs (of any breed) to test the effect of 10 weeks of rapamycin feeding on ageing outcomes particularly cardiac function [82]. It is hoped that this small scale pilot 
study will be extended to a larger trial with power to determine if the drug reduces neoplastic and kidney disease and cognitive decline with age. The value of this model lies partly in the extensive health measures obtainable as pet dogs have access to veterinary services as sophisticated as human medicine, and also that the dogs share the human environment.

\section{Non-human primates}

A research gold-standard for interventions that impact on longevity and healthspan in humans is testing on very closely related non-human primates. This is fraught with ethical issues as well as the practical ones of extremely long lifespans requiring longitudinal studies. While not widely used in ageing research per se (studies focus more on brain function and vaccine trials), two landmark longitudinal studies in primates have assessed the impact of caloric restriction on ageing over a period of nearly 35 years $[83,84]$. Despite differences in reported outcomes (that probably reflect initial health of the monkeys as well as differences in their 'ad lib' diets), it appears that reduction in ad lib food intake by $30 \%$ over the lifetime of an animal leads to highly significant gains in health measures including improved muscle strength, cognitive function and overall lifespan ([83-85]. Caloric restriction, which shows benefit to yeast cells, is therefore also relevant to worms, flies, mice and monkeys (reviewed in $[65,86])$ : there are currently human populations imposing caloric restriction regimes in which surrogate markers of health (e.g. low inflammatory markers) suggest benefit [87]. However, even though non-human primates are genetically and anatomically extremely closely related to humans, significant metabolic differences are emerging that may play a role in rates of ageing. For example, humans burn more calories per unit body weight than chimps, and consequently are more likely to have greater fat stores to cope with this additional energy need [88]; since fat deposits are proinflammatory and inflammation contributes to ageing, it may only be relevant to study such aspects in humans.

\section{Humans}

\section{Human cells and cell senescence}

Primary human cells grown in culture have proven to be extremely useful models for analysis of ageing, since they undergo replicative senescence after multiple rounds of cell division, which results in gradual loss of telomeric DNA until telomere attrition triggers a DNA damage response. This phenomenon of finite lifespan in culture was first described by 
Leonard Hayflick [89] and is now termed the Hayflick limit. Such senescence in vitro is likely to mimic closely that occurring within human tissues, and occurs through conserved tumour suppressor pathways mediated by $\mathrm{p} 53, \mathrm{Rb}, \mathrm{p} 16$ and $\mathrm{p} 21$ (reviewed in [11]). While telomerase reactivation can rejuvenate mouse tissues [90], it carries with an inherent cancer risk [91]. Telomere attrition is by no means the only route to cellular senescence. Other stresses can also trigger senescence, including oncogene activation, DNA damage, ER stress and oxidative stress (reviewed [10]. It was by studying cell senescence in vitro that the SASP was identified [92], and the ability of senescent cells to induce senescence in surrounding cells [93], both through secreted SASP factors and potentially through direct cell-cell contacts [94].

\section{Premature ageing syndromes}

Human premature ageing syndromes provide a system for studying the impact of individual genes on human ageing. In particular, Hutchinson Gilford progeria syndrome (HGPS), a severe early-onset premature ageing syndrome and adult onset Werner syndrome (WS) have both provided insights into mechanisms of ageing (reviewed [95]). While caused by single gene mutations (LMNA in HGPS and WRN in WS), these syndrome show many signs and diseases of normal ageing. Children with HGPS suffer with arthritis and lipodystrophy, with severe arterial intimal thickening resulting in often fatal cardiovascular events; all these changes results from incorrect retention of a pre-lamin protein called progerin in the nuclear envelope, which impacts on gene expression patterns and genome stability [96, 97]. WRN, by contrast, is a helicase/nuclease implicated in maintaining DNA stability during DNA replication and recombination - it is also thought to be involved in some forms of DNA repair. WS patients show a high incidence of sarcomas, while WRN gene promoter inactivation by methylation is implicated in colorectal and other cancers [98]. Notably in terms of ageing, one WRN polymorphism has been suggested to be more prevalent in Finnish centenarians [99]. Recent studies in human induced pluripotent stem cells (iPSCs) with mutant WRN have suggested that marked changed occur in chromatin organisation once cells have undergone the epithelial to mesenchymal transition during differentiation, which leads to differences in gene expression patterns predisposing to ageing [100]. Moreover, the DNA damage arising from WRN loss both in the global genome and at telomeres, and through general nuclear instability in HGPS, is likely to trigger premature 
cellular senescence leading to a failure of tissue regeneration and premature stem cell exhaustion [101]. Progerin-driven cardiovascular disease is not restricted to children with HGPS but also observed with increasing age in the wider population [102]. Drugs such as farnesyl transferase inhibitors that can help to treat aspects of HGPS [103] are therefore likely to be useful in promoting cardiovascular health in later life. Hence monogenic premature ageing disorders can be informative of processes likely to occur in normal ageing.

\section{Longitudinal human studies}

Studies of 'normal' ageing in humans through large scale longitudinal studies (e.g. the Lothian birth cohort studies [104]; Framingham heart study [105] and the Newcastle 85+ project https://www.mrc.ac.uk/research/facilities/cohort-directory/newcastle-85-study) are now

yielding extremely informative data. The emergence of -omics techniques for analysis of the entire genome (genomics), gene expression patterns (transcriptomics), protein complement (proteomics) or metabolic activities of cells (metabolomics) from longitudinal study participants, coupled with non-invasive imaging techniques such as white/grey matter brain MRI [106] and other measures such as hand grip strength and chair rising time, together with standard tests of cognitive function, permit genotype to phenotype correlations in these populations (e.g. [107]). Additional even larger human population studies are underway, such as the UK Biobank project [108] which promises to provide rich resources for identifying biomarkers and other factors that correlate with healthy ageing [109].

\section{Human genes that influence ageing - genome-wide association studies}

Many genome-wide association studies have been carried out in an attempt to identify genes that may promote human longevity. Analysis of a genetically homogeneous Ashkenazi Jewish group of super-centenarians (aged 95-112) taking part in the Longevity Genes Project highlighted four genes that predispose not only to extreme long life, but improved health. The key genes identified in this study are listed in Table 2; perhaps unsurprisingly, several are associated with lipid metabolism conferring cardiovascular and central nervous system, protection.

\section{Epigenetic modulators of ageing}

While inheritance of specific alleles of a few key genes can promote longevity, it is likely that there are multiple other modulators of ageing, each of which is likely to have small but 
additive effects and which may account for inherited longevity even in the absence of the few known favourable longevity alleles (Table 2).

Epigenetic factors regulate gene expression patterns come into play and recent studies in $C$. elegans have demonstrated that chromatin modifiers can have a highly significant impact on lifespan, presumably through modulating expression of the key pathways implicated in ageing [110]. Furthermore, premature ageing is associated with marked charges in the chromatin landscape likely to impact on gene expression (see above): until we have a complete map of the epigenome and an idea of levels of gene expression (mRNAs, miR, and IncRNAs) together with functionality of protein products, we are unlikely to be able to dissect all the features that contribute to ageing. Methylation of the DNA has been reported to provide the best predictor of all-cause mortality [111] - hence we suggest that it is not simply the DNA sequence that should be examined in GWAS studies, but the state of the genome in terms of epigenetic modifications, particularly CpG methylation status, as measured in the Lothian cohort studies [112].

\section{Stress and human ageing}

The role of stress on ageing has been the subject of intense study. Since telomere attrition is linked with replicative senescence, it is notable that peripheral blood cells from mothers caring for severely disabled children (hence experiencing chronic psychosocial stress) were shorter than those without the caring stresses [113]. Furthermore, telomere shortening can be detected in children exposed to domestic violence [114] and longitudinal studies suggest that children from deprived backgrounds age less well than their peers of higher socioeconomic status (though no link between telomere length and ageing has been reported in the cohort studies [115]). Levels of immunomodulatory stress hormones cortisol and DHEA alter on ageing; circadian rhythm changes in hormone levels have recently been exploited clinically to maximise immune responsiveness to flu vaccination in the elderly [116]. Human emotions can also impact on rates of ageing, and various studies have shown that optimistic personality traits predispose to greater longevity [117], while depression increases the risk of death e.g. after falls in the elderly [118]. Such studies are impossible to conduct in animal models, requiring as they do questionnaires of mood and personality traits. Thus any anti-ageing agents developed in the lab must be administered in the context 
of a complex human comprising not only the physical but also the emotional person. It will also be critical to consider the interplay between clinical interventions (especially with drugs or dietary supplements) and the microbiome, constituting as it does $\sim 90 \%$ of the cells of an adult human and influencing inflammation, gut permeability and even cancer risks.

\section{Conclusions}

Though human ageing manifests through apparently disparate diseases currently treated as separate entities, it is likely that there is a common underlying cause of age-related disease and frailty that can be traced back to the way in which cells deal with environmental and endogenous insult over time by entering the state of cellular senescence [12]. The study of ageing has recently been accelerated by the development of new tools that permit analysis of the genome, epigenome, transcriptome, proteome and metabolome of old cells and tissues, and that have highlighted major changes in senescent cells that are now being exploited for development of possible therapies aiming either to modify deleterious aspects of senescent cells (seno-therapeutics) or kill senescent cells outright (senolytics). That senescent cells are a causative feature of ageing is becoming clear from experiments using senolytic drugs in mice. Model systems have contributed hugely to our understanding of key biochemical pathways of ageing and highlighted novel targets for drug development. However, such studies cannot take into account the cross-talk between emotional and social state and physical ageing - social isolation, for example, is a known contributor to unhealthy ageing (e.g. [119]). Hence we propose that an interdisciplinary approach integrating biochemistry, model organism studies and human trials are needed in order to best assess which interventions have greatest promise in alleviating diseases and frailties to promote healthier ageing in human populations.

One such human trial in progress is TAME, (targeting aging with metformin) with mixed endpoints of delay in onset of various different age-related pathologies ${ }^{1}$. In a single endpoint trial on ageing immunity, Mannick and colleagues showed that short-term treatment with RAD001 (also known as everolimus, a rapamycin-like mTORC1 inhibitor) prior to flu vaccination was beneficial in increasing not only anti-flu antibody titres but also in reducing expression of the PD death receptor in B and T cells, thus alleviating immune

\footnotetext{
${ }^{1}$ http://www.afar.org/natgeos
} 
senescence [120]. Hence small-scale trials of drugs already approved by the FDA are currently taking place for indications of ageing such as immune senescence or delayed disease onset; this is likely to establish a precedent allowing novel agents identified by screening in model organisms to progress to human trials as possible therapies for agerelated indications.

\section{Acknowledgements}

We gratefully acknowledge the generous financial support of an anonymous donor (via the University of Oxford Legacies Office) supporting the work of HW, and to the Glenn Foundation for Medical Research for an award to LSC. HL is funded though BBSRC grant number [BB/M006727/1] jointly to LSC and Prof Alison Woollard.

\section{References:}

[1] R. Crawford, C. Emmerson, G. Tetlow, A survey of public spending in the UK Institute of Fiscal Studies, 2009.

[2] C. Lopez-Otin, M.A. Blasco, L. Partridge, M. Serrano, G. Kroemer, The hallmarks of aging, Cell 153(6) (2013) 1194-217.

[3] N.F. Mathon, A.C. Lloyd, Milestones in cell division : Cell senescence and cancer, Nat Rev Cancer 1(3) (2001) 203-213.

[4] K.A. Cho, S.J. Ryu, Y.S. Oh, J.H. Park, J.W. Lee, H.-P. Kim, K.T. Kim, I.S. Jang, S.C. Park, Morphological Adjustment of Senescent Cells by Modulating Caveolin-1 Status, Journal of Biological Chemistry 279(40) (2004) 42270-42278.

[5] L. Garcia-Prat, M. Martinez-Vicente, E. Perdiguero, L. Ortet, J. Rodriguez-Ubreva, E. Rebollo, V. Ruiz-Bonilla, S. Gutarra, E. Ballestar, A.L. Serrano, M. Sandri, P. Munoz-Canoves, Autophagy maintains stemness by preventing senescence, Nature 529(7584) (2016) 37-42. [6] G.P. Dimri, X. Lee, G. Basile, M. Acosta, G. Scott, C. Roskelley, E.E. Medrano, M. Linskens, I. Rubelj, O. Pereira-Smith, et al., A biomarker that identifies senescent human cells in culture and in aging skin in vivo, Proc Natl Acad Sci U S A 92(20) (1995) 9363-7.

[7] E.L. James, R.D. Michalek, G.N. Pitiyage, A.M. de Castro, K.S. Vignola, J. Jones, R.P. Mohney, E.D. Karoly, S.S. Prime, E.K. Parkinson, Senescent human fibroblasts show increased glycolysis and redox homeostasis with extracellular metabolomes that overlap with those of irreparable DNA damage, aging, and disease, Journal of proteome research 14(4) (2015) 1854-71.

[8] H.C. Lee, P.H. Yin, C.W. Chi, Y.H. Wei, Increase in mitochondrial mass in human fibroblasts under oxidative stress and during replicative cell senescence, Journal of biomedical science 9(6 Pt 1) (2002) 517-26.

[9] D.J. Colacurcio, R.A. Nixon, Disorders of lysosomal acidification-the emerging role of vATPase in aging and neurodegenerative disease, Ageing research reviews (2016). [10] J.M. van Deursen, The role of senescent cells in ageing, Nature 509(7501) (2014) 439- 
46.

[11] D. Munoz-Espin, M. Serrano, Cellular senescence: from physiology to pathology, Nat Rev Mol Cell Biol 15(7) (2014) 482-96.

[12] L.S. Cox, P.A. Mason, M.C. Bagley, D. Steinsaltz, A. Stefanovska, A. Bernjak, P.V.E. McClintock, A.C. Phillips, J. Upton, J.E. Latimer, T. Davis, Understanding ageing: biological and social perspectives, in: A. Walker (Ed.), The new science of ageing, Policy Press, Bristol, 2014, pp. 25-75.

[13] W. Klapper, K. Kuhne, K.K. Singh, K. Heidorn, R. Parwaresch, G. Krupp, Longevity of lobsters is linked to ubiquitous telomerase expression, FEBS letters 439(1-2) (1998) 143-6.

[14] R. Schaible, A. Scheuerlein, M.J. Danko, J. Gampe, D.E. Martinez, J.W. Vaupel, Constant mortality and fertility over age in Hydra, Proc Natl Acad Sci U S A 112(51) (2015) 15701-6.

[15] E.E. Philipp, D. Abele, Masters of longevity: lessons from long-lived bivalves--a minireview, Gerontology 56(1) (2010) 55-65.

[16] G. Eguchi, Y. Eguchi, K. Nakamura, M.C. Yadav, J.L. Millan, P.A. Tsonis, Regenerative capacity in newts is not altered by repeated regeneration and ageing, Nature communications 2 (2011) 384.

[17] M.H. Yun, H. Davaapil, J.P. Brockes, Recurrent turnover of senescent cells during regeneration of a complex structure, eLife 4 (2015).

[18] J. Campisi, Aging and cancer: the double-edged sword of replicative senescence, Journal of the American Geriatrics Society 45(4) (1997) 482-8.

[19] Saccharomyces genome database. <http://www.yeastgenome.org/>.

[20] Wormbase. <http://www.wormbase.org>.

[21] Flybase. <http://flybase.org/>.

[22] T.F. Outeiro, S. Lindquist, Yeast cells provide insight into alpha-synuclein biology and pathobiology, Science 302(5651) (2003) 1772-5.

[23] P. Zabrocki, K. Pellens, T. Vanhelmont, T. Vandebroek, G. Griffioen, S. Wera, F. Van Leuven, J. Winderickx, Characterization of alpha-synuclein aggregation and synergistic toxicity with protein tau in yeast, FEBS J 272(6) (2005) 1386-400.

[24] A.B. Meriin, X. Zhang, X. He, G.P. Newnam, Y.O. Chernoff, M.Y. Sherman, Huntington toxicity in yeast model depends on polyglutamine aggregation mediated by a prion-like protein Rnq1, J Cell Biol 157(6) (2002) 997-1004.

[25] M. Kaeberlein, K.T. Kirkland, S. Fields, B.K. Kennedy, Genes determining yeast replicative life span in a long-lived genetic background, Mech Ageing Dev 126(4) (2005) 491504.

[26] M. Kaeberlein, R.W. Powers, 3rd, K.K. Steffen, E.A. Westman, D. Hu, N. Dang, E.O. Kerr, K.T. Kirkland, S. Fields, B.K. Kennedy, Regulation of yeast replicative life span by TOR and Sch9 in response to nutrients, Science 310(5751) (2005) 1193-6.

[27] M. Wei, P. Fabrizio, J. Hu, H. Ge, C. Cheng, L. Li, V.D. Longo, Life span extension by calorie restriction depends on Rim15 and transcription factors downstream of Ras/PKA, Tor, and Sch9, PLoS genetics 4(1) (2008) e13.

[28] A. Soulard, A. Cremonesi, S. Moes, F. Schutz, P. Jeno, M.N. Hall, The rapamycin-sensitive phosphoproteome reveals that TOR controls protein kinase $A$ toward some but not all substrates, Mol Biol Cell 21(19) (2010) 3475-86.

[29] Y. Tian, C. Luo, Q. Ouyang, A microfluidic synchronizer for fission yeast cells, Lab Chip 13(20) (2013) 4071-7.

[30] A. Baryshnikova, M. Costanzo, Y. Kim, H. Ding, J. Koh, K. Toufighi, J.Y. Youn, J. Ou, B.J. San Luis, S. Bandyopadhyay, M. Hibbs, D. Hess, A.C. Gingras, G.D. Bader, O.G. Troyanskaya, 
G.W. Brown, B. Andrews, C. Boone, C.L. Myers, Quantitative analysis of fitness and genetic interactions in yeast on a genome scale, Nat Methods 7(12) (2010) 1017-24.

[31] M.R. Klass, Aging in the nematode Caenorhabditis elegans: major biological and environmental factors influencing life span, Mech Ageing Dev 6(6) (1977) 413-29.

[32] Consortium, Genome sequence of the nematode C. elegans: a platform for investigating biology, Science 282(5396) (1998) 2012-8.

[33] R. Baumeister, L. Ge, The worm in us - Caenorhabditis elegans as a model of human disease, Trends Biotechnol 20(4) (2002) 147-8.

[34] M.R. Klass, A method for the isolation of longevity mutants in the nematode Caenorhabditis elegans and initial results, Mech Ageing Dev 22(3-4) (1983) 279-86.

[35] D.B. Friedman, T.E. Johnson, A mutation in the age-1 gene in Caenorhabditis elegans lengthens life and reduces hermaphrodite fertility, Genetics 118(1) (1988) 75-86.

[36] J.Z. Morris, H.A. Tissenbaum, G. Ruvkun, A phosphatidylinositol-3-OH kinase family member regulating longevity and diapause in Caenorhabditis elegans, Nature 382(6591) (1996) 536-9.

[37] M. Barbieri, M. Bonafe, C. Franceschi, G. Paolisso, Insulin/IGF-I-signaling pathway: an evolutionarily conserved mechanism of longevity from yeast to humans, Am J Physiol Endocrinol Metab 285(5) (2003) E1064-71.

[38] K. Yen, S.D. Narasimhan, H.A. Tissenbaum, DAF-16/Forkhead box O transcription factor: many paths to a single Fork(head) in the road, Antioxid Redox Signal 14(4) (2011) 623-34. [39] K. Lin, J.B. Dorman, A. Rodan, C. Kenyon, daf-16: An HNF-3/forkhead family member that can function to double the life-span of Caenorhabditis elegans, Science 278(5341) (1997) 1319-22.

[40] S. Ogg, S. Paradis, S. Gottlieb, G.I. Patterson, L. Lee, H.A. Tissenbaum, G. Ruvkun, The Fork head transcription factor DAF-16 transduces insulin-like metabolic and longevity signals in C. elegans, Nature 389(6654) (1997) 994-9.

[41] K. Banasik, R. Ribel-Madsen, A.P. Gjesing, L. Wegner, A. Andersson, P. Poulsen, A. Borglykke, D.R. Witte, O. Pedersen, T. Hansen, A. Vaag, The FOXO3A rs2802292 G-allele associates with improved peripheral and hepatic insulin sensitivity and increased skeletal muscle-FOXO3A mRNA expression in twins, J Clin Endocrinol Metab 96(1) (2011) E119-24. [42] K.L. Lunetta, R.B. D'Agostino, Sr., D. Karasik, E.J. Benjamin, C.Y. Guo, R. Govindaraju, D.P. Kiel, M. Kelly-Hayes, J.M. Massaro, M.J. Pencina, S. Seshadri, J.M. Murabito, Genetic correlates of longevity and selected age-related phenotypes: a genome-wide association study in the Framingham Study, BMC Med Genet 8 Suppl 1 (2007) S13.

[43] B.J. Willcox, T.A. Donlon, Q. He, R. Chen, J.S. Grove, K. Yano, K.H. Masaki, D.C. Willcox, B. Rodriguez, J.D. Curb, FOXO3A genotype is strongly associated with human longevity, Proc Natl Acad Sci U S A 105(37) (2008) 13987-92.

[44] Y. Zeng, L. Cheng, H. Chen, H. Cao, E.R. Hauser, Y. Liu, Z. Xiao, Q. Tan, X.L. Tian, J.W. Vaupel, Effects of FOXO genotypes on longevity: a biodemographic analysis, J Gerontol A Biol Sci Med Sci 65(12) (2010) 1285-99.

[45] F. Flachsbart, A. Caliebe, R. Kleindorp, H. Blanche, H. von Eller-Eberstein, S. Nikolaus, S. Schreiber, A. Nebel, Association of FOXO3A variation with human longevity confirmed in German centenarians, Proc Natl Acad Sci U S A 106(8) (2009) 2700-5.

[46] H.A. Tissenbaum, Using for aging research, Invertebr Reprod Dev 59(sup1) (2015) 5963.

[47] A. Olsen, M.C. Vantipalli, G.J. Lithgow, Using Caenorhabditis elegans as a model for aging and age-related diseases, Ann N Y Acad Sci 1067 (2006) 120-8. 
[48] D.S. Fay, A. Fluet, C.J. Johnson, C.D. Link, In vivo aggregation of beta-amyloid peptide variants, J Neurochem 71(4) (1998) 1616-25.

[49] C.D. Link, C.J. Johnson, Reporter transgenes for study of oxidant stress in Caenorhabditis elegans, Methods Enzymol 353 (2002) 497-505.

[50] R. Nass, D.H. Hall, D.M. Miller, 3rd, R.D. Blakely, Neurotoxin-induced degeneration of dopamine neurons in Caenorhabditis elegans, Proc Natl Acad Sci U S A 99(5) (2002) 3264-9. [51] M.J. Gravato-Nobre, H.R. Nicholas, R. Nijland, D. O'Rourke, D.E. Whittington, K.J. Yook, J. Hodgkin, Multiple Genes Affect Sensitivity of Caenorhabditis elegans to the Bacterial Pathogen Microbacterium nematophilum, Genetics 171(3) (2005) 1033-45.

[52] S. Chien, L.T. Reiter, E. Bier, M. Gribskov, Homophila: human disease gene cognates in Drosophila, Nucleic Acids Res 30(1) (2002) 149-51.

[53] M. Waskar, Y. Li, J. Tower, Stem cell aging in the Drosophila ovary, Age (Dordr) 27(3) (2005) 201-12.

[54] D.J. Clancy, D. Gems, L.G. Harshman, S. Oldham, H. Stocker, E. Hafen, S.J. Leevers, L. Partridge, Extension of life-span by loss of CHICO, a Drosophila insulin receptor substrate protein, Science 292(5514) (2001) 104-6.

[55] P. Kapahi, B.M. Zid, T. Harper, D. Koslover, V. Sapin, S. Benzer, Regulation of lifespan in Drosophila by modulation of genes in the TOR signaling pathway, Curr Biol 14(10) (2004) 885-90.

[56] I. Bjedov, J.M. Toivonen, F. Kerr, C. Slack, J. Jacobson, A. Foley, L. Partridge, Mechanisms of life span extension by rapamycin in the fruit fly Drosophila melanogaster, Cell Metab 11(1) 35-46.

[57] L.E. Gimenez, P. Ghildyal, K.E. Fischer, H. Hu, W.W. Ja, B.A. Eaton, Y. Wu, S.N. Austad, R. Ranjan, Modulation of methuselah expression targeted to Drosophila insulin-producing cells extends life and enhances oxidative stress resistance, Aging Cell 12(1) (2012) 121-9.

[58] M. Rera, R.I. Clark, D.W. Walker, Intestinal barrier dysfunction links metabolic and inflammatory markers of aging to death in Drosophila, Proc Natl Acad Sci U S A 109(52) (2012) 21528-33.

[59] G. Gasque, S. Conway, J. Huang, Y. Rao, L.B. Vosshall, Small molecule drug screening in Drosophila identifies the $5 \mathrm{HT} 2 \mathrm{~A}$ receptor as a feeding modulation target, Scientific reports 3 (2013) srep02120.

[60] R.D. Saunders, I. Boubriak, D.J. Clancy, L.S. Cox, Identification and characterization of a Drosophila ortholog of WRN exonuclease that is required to maintain genome integrity, Aging Cell 7(3) (2008) 418-25.

[61] M.D. Rand, A.L. Kearney, J. Dao, T. Clason, Permeabilization of Drosophila embryos for introduction of small molecules, Insect Biochem Mol Biol 40(11) (2010) 792-804.

[62] J.M. Zahn, S. Poosala, A.B. Owen, D.K. Ingram, A. Lustig, A. Carter, A.T. Weeraratna, D.D. Taub, M. Gorospe, K. Mazan-Mamczarz, E.G. Lakatta, K.R. Boheler, X. Xu, M.P. Mattson, G. Falco, M.S. Ko, D. Schlessinger, J. Firman, S.K. Kummerfeld, W.H. Wood, 3rd, A.B.

Zonderman, S.K. Kim, K.G. Becker, AGEMAP: a gene expression database for aging in mice, PLoS genetics 3(11) (2007) e201.

[63] A.L. Duran, P. Potter, S. Wells, T. Kirkwood, T. von Zglinicki, A. McArdle, C. Scudamore, Q.J. Meng, G. de Haan, A. Corcoran, I. Bellantuono, Shared Ageing Research Models (ShARM): a new facility to support ageing research, Biogerontology 14(6) (2013) 789-94. [64] J.O. Hill, A. Latiff, M. DiGirolamo, Effects of variable caloric restriction on utilization of ingested energy in rats, Am J Physiol 248(5 Pt 2) (1985) R549-59.

[65] L.S. Cox, J.A. Mattison, Increasing longevity through caloric restriction or rapamycin 
feeding in mammals: common mechanisms for common outcomes?, Aging Cell 8(5) (2009) 607-13.

[66] D.E. Harrison, R. Strong, Z.D. Sharp, J.F. Nelson, C.M. Astle, K. Flurkey, N.L. Nadon, J.E. Wilkinson, K. Frenkel, C.S. Carter, M. Pahor, M.A. Javors, E. Fernandez, R.A. Miller, Rapamycin fed late in life extends lifespan in genetically heterogeneous mice, Nature 460(7253) (2009) 392-5.

[67] R.A. Miller, D.E. Harrison, C.M. Astle, J.A. Baur, A.R. Boyd, R. de Cabo, E. Fernandez, K. Flurkey, M.A. Javors, J.F. Nelson, C.J. Orihuela, S. Pletcher, Z.D. Sharp, D. Sinclair, J.W.

Starnes, J.E. Wilkinson, N.L. Nadon, R. Strong, Rapamycin, but not resveratrol or simvastatin, extends life span of genetically heterogeneous mice, J Gerontol A Biol Sci Med Sci 66(2) (2011) 191-201.

[68] A. Caccamo, S. Majumder, A. Richardson, R. Strong, S. Oddo, Molecular interplay between mammalian target of rapamycin (mTOR), amyloid-beta, and Tau: effects on cognitive impairments, J Biol Chem 285(17) (2010) 13107-20.

[69] P. Spilman, N. Podlutskaya, M.J. Hart, J. Debnath, O. Gorostiza, D. Bredesen, A. Richardson, R. Strong, V. Galvan, Inhibition of mTOR by rapamycin abolishes cognitive deficits and reduces amyloid-beta levels in a mouse model of Alzheimer's disease, PLoS One 5(4) (2010) e9979.

[70] S. Majumder, A. Richardson, R. Strong, S. Oddo, Inducing autophagy by rapamycin before, but not after, the formation of plaques and tangles ameliorates cognitive deficits, PLoS One 6(9) (2011) e25416.

[71] D.J. Puleston, H. Zhang, T.J. Powell, E. Lipina, S. Sims, I. Panse, A.S. Watson, V. Cerundolo, A.R. Townsend, P. Klenerman, A.K. Simon, Autophagy is a critical regulator of memory CD8(+) T cell formation, eLife 3 (2014).

[72] F.S. Wyllie, C.J. Jones, J.W. Skinner, M.F. Haughton, C. Wallis, D. Wynford-Thomas, R.G. Faragher, D. Kipling, Telomerase prevents the accelerated cell ageing of Werner syndrome fibroblasts, Nature genetics 24(1) (2000) 16-7.

[73] C. Pantoja, M. Serrano, Murine fibroblasts lacking p21 undergo senescence and are resistant to transformation by oncogenic Ras, Oncogene 18(35) (1999) 4974-82.

[74] S.D. Tyner, S. Venkatachalam, J. Choi, S. Jones, N. Ghebranious, H. Igelmann, X. Lu, G. Soron, B. Cooper, C. Brayton, S.H. Park, T. Thompson, G. Karsenty, A. Bradley, L.A. Donehower, p53 mutant mice that display early ageing-associated phenotypes, Nature 415(6867) (2002) 45-53.

[75] D.J. Baker, T. Wijshake, T. Tchkonia, N.K. LeBrasseur, B.G. Childs, B. van de Sluis, J.L. Kirkland, J.M. van Deursen, Clearance of p16Ink4a-positive senescent cells delays ageingassociated disorders, Nature 479(7372) (2011) 232-6.

[76] D.J. Baker, B.G. Childs, M. Durik, M.E. Wijers, C.J. Sieben, J. Zhong, R.A. Saltness, K.B. Jeganathan, G.C. Verzosa, A. Pezeshki, K. Khazaie, J.D. Miller, J.M. van Deursen, Naturally occurring p16(Ink4a)-positive cells shorten healthy lifespan, Nature 530(7589) (2016) 184-9. [77] J. Chang, Y. Wang, L. Shao, R.M. Laberge, M. Demaria, J. Campisi, K. Janakiraman, N.E. Sharpless, S. Ding, W. Feng, Y. Luo, X. Wang, N. Aykin-Burns, K. Krager, U. Ponnappan, M. Hauer-Jensen, A. Meng, D. Zhou, Clearance of senescent cells by ABT263 rejuvenates aged hematopoietic stem cells in mice, Nat Med 22(1) (2015) 78-83.

[78] Y. Zhu, T. Tchkonia, T. Pirtskhalava, A.C. Gower, H. Ding, N. Giorgadze, A.K. Palmer, Y. Ikeno, G.B. Hubbard, M. Lenburg, S.P. O'Hara, N.F. LaRusso, J.D. Miller, C.M. Roos, G.C. Verzosa, N.K. LeBrasseur, J.D. Wren, J.N. Farr, S. Khosla, M.B. Stout, S.J. McGowan, H. Fuhrmann-Stroissnigg, A.U. Gurkar, J. Zhao, D. Colangelo, A. Dorronsoro, Y.Y. Ling, A.S. 
Barghouthy, D.C. Navarro, T. Sano, P.D. Robbins, L.J. Niedernhofer, J.L. Kirkland, The Achilles' heel of senescent cells: from transcriptome to senolytic drugs, Aging Cell 14(4) (2015) 644-58.

[79] J.C. Triplett, A. Tramutola, A. Swomley, J. Kirk, K. Grimes, K. Lewis, M. Orr, K. Rodriguez, J. Cai, J.B. Klein, M. Perluigi, R. Buffenstein, D.A. Butterfield, Age-related changes in the proteostasis network in the brain of the naked mole-rat: Implications promoting healthy longevity, Biochim Biophys Acta 1852(10 Pt A) (2015) 2213-24.

[80] X. Tian, J. Azpurua, Z. Ke, A. Augereau, Z.D. Zhang, J. Vijg, V.N. Gladyshev, V. Gorbunova, A. Seluanov, INK4 locus of the tumor-resistant rodent, the naked mole rat, expresses a functional p15/p16 hybrid isoform, Proc Natl Acad Sci U S A 112(4) (2015) 1053-8.

[81] X. Tian, J. Azpurua, C. Hine, A. Vaidya, M. Myakishev-Rempel, J. Ablaeva, Z. Mao, E. Nevo, V. Gorbunova, A. Seluanov, High-molecular-mass hyaluronan mediates the cancer resistance of the naked mole rat, Nature 499(7458) (2013) 346-9.

[82] M. Kaeberlein, K.E. Creevy, D.E. Promislow, The dog aging project: translational geroscience in companion animals, Mamm Genome 3 (2016) 3.

[83] R.J. Colman, R.M. Anderson, S.C. Johnson, E.K. Kastman, K.J. Kosmatka, T.M. Beasley, D.B. Allison, C. Cruzen, H.A. Simmons, J.W. Kemnitz, R. Weindruch, Caloric restriction delays disease onset and mortality in rhesus monkeys, Science 325(5937) (2009) 201-4.

[84] J.A. Mattison, G.S. Roth, T.M. Beasley, E.M. Tilmont, A.M. Handy, R.L. Herbert, D.L. Longo, D.B. Allison, J.E. Young, M. Bryant, D. Barnard, W.F. Ward, W. Qi, D.K. Ingram, R. de Cabo, Impact of caloric restriction on health and survival in rhesus monkeys from the NIA study, Nature 489(7415) (2012) 318-21.

[85] R.J. Colman, T.M. Beasley, J.W. Kemnitz, S.C. Johnson, R. Weindruch, R.M. Anderson, Caloric restriction reduces age-related and all-cause mortality in rhesus monkeys, Nature communications 5 (2014) 3557.

[86] L.S. Cox, Live fast, die young: new lessons in mammalian longevity, Rejuvenation Res 12(4) (2009) 283-8.

[87] J.O. Holloszy, L. Fontana, Caloric restriction in humans, Exp Gerontol 42(8) (2007) 70912.

[88] A. Gibbons, HUMAN EVOLUTION. Why humans are the high-energy apes, Science 352(6286) (2016) 639.

[89] L. Hayflick, P.S. Moorhead, The serial cultivation of human diploid cell strains, Exp Cell Res 25 (1961) 585-621.

[90] M. Jaskelioff, F.L. Muller, J.H. Paik, E. Thomas, S. Jiang, A.C. Adams, E. Sahin, M. KostAlimova, A. Protopopov, J. Cadinanos, J.W. Horner, E. Maratos-Flier, R.A. Depinho,

Telomerase reactivation reverses tissue degeneration in aged telomerase-deficient mice, Nature 469(7328) (2010) 102-6.

[91] Z. Ding, C.J. Wu, M. Jaskelioff, E. Ivanova, M. Kost-Alimova, A. Protopopov, G.C. Chu, G. Wang, X. Lu, E.S. Labrot, J. Hu, W. Wang, Y. Xiao, H. Zhang, J. Zhang, B. Gan, S.R. Perry, S. Jiang, L. Li, J.W. Horner, Y.A. Wang, L. Chin, R.A. DePinho, Telomerase reactivation following telomere dysfunction yields murine prostate tumors with bone metastases, Cell 148(5) (2012) 896-907.

[92] F. Rodier, J.P. Coppe, C.K. Patil, W.A. Hoeijmakers, D.P. Munoz, S.R. Raza, A. Freund, E. Campeau, A.R. Davalos, J. Campisi, Persistent DNA damage signalling triggers senescenceassociated inflammatory cytokine secretion, Nat Cell Biol 11(8) (2009) 973-9.

[93] G. Nelson, J. Wordsworth, C. Wang, D. Jurk, C. Lawless, C. Martin-Ruiz, T. von Zglinicki, A senescent cell bystander effect: senescence-induced senescence, Aging Cell 11(2) (2012) 
345-9.

[94] A. Biran, M. Perelmutter, H. Gal, D.G. Burton, Y. Ovadya, E. Vadai, T. Geiger, V.

Krizhanovsky, Senescent cells communicate via intercellular protein transfer, Genes \& development 29(8) (2015) 791-802.

[95] L.S. Cox, R.G. Faragher, From old organisms to new molecules: integrative biology and therapeutic targets in accelerated human ageing, Cellular and molecular life sciences: CMLS 64(19-20) (2007) 2620-41.

[96] M. Eriksson, W.T. Brown, L.B. Gordon, M.W. Glynn, J. Singer, L. Scott, M.R. Erdos, C.M. Robbins, T.Y. Moses, P. Berglund, A. Dutra, E. Pak, S. Durkin, A.B. Csoka, M. Boehnke, T.W. Glover, F.S. Collins, Recurrent de novo point mutations in lamin A cause Hutchinson-Gilford progeria syndrome, Nature 423(6937) (2003) 293-8.

[97] D.K. Shumaker, T. Dechat, A. Kohlmaier, S.A. Adam, M.R. Bozovsky, M.R. Erdos, M. Eriksson, A.E. Goldman, S. Khuon, F.S. Collins, T. Jenuwein, R.D. Goldman, Mutant nuclear lamin A leads to progressive alterations of epigenetic control in premature aging, Proc Natl Acad Sci U S A 103(23) (2006) 8703-8.

[98] R. Agrelo, W.H. Cheng, F. Setien, S. Ropero, J. Espada, M.F. Fraga, M. Herranz, M.F. Paz, M. Sanchez-Cespedes, M.J. Artiga, D. Guerrero, A. Castells, C. von Kobbe, V.A. Bohr, M. Esteller, Epigenetic inactivation of the premature aging Werner syndrome gene in human cancer, Proc Natl Acad Sci U S A 103(23) (2006) 8822-7.

[99] E. Castro, C.E. Ogburn, K.E. Hunt, R. Tilvis, J. Louhija, R. Penttinen, R. Erkkola, A. Panduro, R. Riestra, C. Piussan, S.S. Deeb, L. Wang, S.D. Edland, G.M. Martin, J. Oshima, Polymorphisms at the Werner locus: I. Newly identified polymorphisms, ethnic variability of 1367Cys/Arg, and its stability in a population of Finnish centenarians, Am J Med Genet 82(5) (1999) 399-403.

[100] W. Zhang, J. Li, K. Suzuki, J. Qu, P. Wang, J. Zhou, X. Liu, R. Ren, X. Xu, A. Ocampo, T. Yuan, J. Yang, Y. Li, L. Shi, D. Guan, H. Pan, S. Duan, Z. Ding, M. Li, F. Yi, R. Bai, Y. Wang, C. Chen, F. Yang, X. Li, Z. Wang, E. Aizawa, A. Goebl, R.D. Soligalla, P. Reddy, C.R. Esteban, F. Tang, G.H. Liu, J.C. Belmonte, Aging stem cells. A Werner syndrome stem cell model unveils heterochromatin alterations as a driver of human aging, Science 348(6239) (2015) 1160-3. [101] Y. Rosengardten, T. McKenna, D. Grochova, M. Eriksson, Stem cell depletion in Hutchinson-Gilford progeria syndrome, Aging Cell 10(6) (2011) 1011-20.

[102] M. Olive, I. Harten, R. Mitchell, J.K. Beers, K. Djabali, K. Cao, M.R. Erdos, C. Blair, B. Funke, L. Smoot, M. Gerhard-Herman, J.T. Machan, R. Kutys, R. Virmani, F.S. Collins, T.N. Wight, E.G. Nabel, L.B. Gordon, Cardiovascular pathology in Hutchinson-Gilford progeria: correlation with the vascular pathology of aging, Arterioscler Thromb Vasc Biol 30(11) (2010) 2301-9.

[103] L.B. Gordon, J. Massaro, R.B. D'Agostino, Sr., S.E. Campbell, J. Brazier, W.T. Brown, M.E. Kleinman, M.W. Kieran, Impact of farnesylation inhibitors on survival in HutchinsonGilford progeria syndrome, Circulation 130(1) (2014) 27-34.

[104] T.L.B.C.o.a. 1936, <http://www.lothianbirthcohort.ed.ac.uk/>).

[105] F.H. Study, <https://www.framinghamheartstudy.org/>).

[106] B.S. Aribisala, M.C. Valdes Hernandez, N.A. Royle, Z. Morris, S. Munoz Maniega, M.E. Bastin, I.J. Deary, J.M. Wardlaw, Brain atrophy associations with white matter lesions in the ageing brain: the Lothian Birth Cohort 1936, European radiology 23(4) (2013) 1084-92.

[107] S.P. Hagenaars, S.E. Harris, T.K. Clarke, L. Hall, M. Luciano, A.M. Fernandez-Pujals, G. Davies, C. Hayward, J.M. Starr, D.J. Porteous, A.M. McIntosh, I.J. Deary, Polygenic risk for coronary artery disease is associated with cognitive ability in older adults, International 
journal of epidemiology (2016).

[108] R. Collins, What makes UK Biobank special?, The Lancet 379(9822) 1173-1174.

[109] C. Sudlow, J. Gallacher, N. Allen, V. Beral, P. Burton, J. Danesh, P. Downey, P. Elliott, J. Green, M. Landray, B. Liu, P. Matthews, G. Ong, J. Pell, A. Silman, A. Young, T. Sprosen, T. Peakman, R. Collins, UK biobank: an open access resource for identifying the causes of a wide range of complex diseases of middle and old age, PLoS Med 12(3) (2015) e1001779. [110] B.A. Benayoun, E.A. Pollina, A. Brunet, Epigenetic regulation of ageing: linking environmental inputs to genomic stability, Nat Rev Mol Cell Biol 16(10) (2015) 593-610. [111] R.E. Marioni, S. Shah, A.F. McRae, B.H. Chen, E. Colicino, S.E. Harris, J. Gibson, A.K. Henders, P. Redmond, S.R. Cox, A. Pattie, J. Corley, L. Murphy, N.G. Martin, G.W.

Montgomery, A.P. Feinberg, M.D. Fallin, M.L. Multhaup, A.E. Jaffe, R. Joehanes, J. Schwartz, A.C. Just, K.L. Lunetta, J.M. Murabito, J.M. Starr, S. Horvath, A.A. Baccarelli, D. Levy, P.M. Visscher, N.R. Wray, I.J. Deary, DNA methylation age of blood predicts all-cause mortality in later life, Genome Biol 16 (2015) 25.

[112] S. Shah, M.J. Bonder, R.E. Marioni, Z. Zhu, A.F. McRae, A. Zhernakova, S.E. Harris, D. Liewald, A.K. Henders, M.M. Mendelson, C. Liu, R. Joehanes, L. Liang, D. Levy, N.G. Martin, J.M. Starr, C. Wijmenga, N.R. Wray, J. Yang, G.W. Montgomery, L. Franke, I.J. Deary, P.M. Visscher, Improving Phenotypic Prediction by Combining Genetic and Epigenetic Associations, American journal of human genetics 97(1) (2015) 75-85.

[113] X. Chen, J.C. Velez, C. Barbosa, M. Pepper, A. Andrade, L. Stoner, I. De Vivo, B. Gelaye, M.A. Williams, Smoking and perceived stress in relation to short salivary telomere length among caregivers of children with disabilities, Stress 18(1) (2015) 20-8.

[114] I. Shalev, T.E. Moffitt, K. Sugden, B. Williams, R.M. Houts, A. Danese, J. Mill, L. Arseneault, A. Caspi, Exposure to violence during childhood is associated with telomere erosion from 5 to 10 years of age: a longitudinal study, Mol Psychiatry 18(5) (2013) 576-581. [115] S.E. Harris, C. Martin-Ruiz, T. von Zglinicki, J.M. Starr, I.J. Deary, Telomere length and aging biomarkers in 70-year-olds: the Lothian Birth Cohort 1936, Neurobiol Aging 33(7) (2012) 1486 e3-8.

[116] J.E. Long, M.T. Drayson, A.E. Taylor, K.M. Toellner, J.M. Lord, A.C. Phillips, Morning vaccination enhances antibody response over afternoon vaccination: A cluster-randomised trial, Vaccine (2016).

[117] D.D. Danner, D.A. Snowdon, W.V. Friesen, Positive emotions in early life and longevity: findings from the nun study, J Pers Soc Psychol 80(5) (2001) 804-13.

[118] A.C. Phillips, J. Upton, N.A. Duggal, D. Carroll, J.M. Lord, Depression following hip fracture is associated with increased physical frailty in older adults: the role of the cortisol: dehydroepiandrosterone sulphate ratio, BMC Geriatr 13 (2013) 60.

[119] N.K. Valtorta, M. Kanaan, S. Gilbody, S. Ronzi, B. Hanratty, Loneliness and social isolation as risk factors for coronary heart disease and stroke: systematic review and metaanalysis of longitudinal observational studies, Heart (British Cardiac Society) (2016). [120] J.B. Mannick, G. Del Giudice, M. Lattanzi, N.M. Valiante, J. Praestgaard, B. Huang, M.A. Lonetto, H.T. Maecker, J. Kovarik, S. Carson, D.J. Glass, L.B. Klickstein, mTOR inhibition improves immune function in the elderly, Sci Transl Med 6(268) (2014) 268 ra179. 